\title{
Trends in Radiology-Related Medical Lawsuits Identified by a Legal Database Search
}

\author{
Yuki Yamashiro', Hiromichi Kuwabara², Ryohei Kuwatsuru1 \\ ${ }^{1}$ Department of Radiology, Juntendo University, Tokyo, Japan \\ ${ }^{2}$ Department of Hospital Administration, Juntendo University, Tokyo, Japan \\ Email: kuwaturu@juntendo.ac.jp
}

How to cite this paper: Yamashiro, Y., Kuwabara, H. and Kuwatsuru, R. (2018) Trends in Radiology-Related Medical Lawsuits Identified by a Legal Database Search. Open Journal of Radiology, 8, 65-73. https://doi.org/10.4236/ojrad.2018.82008

Received: February 26, 2018

Accepted: June 10, 2018

Published: June 13, 2018

Copyright (c) 2018 by authors and Scientific Research Publishing Inc. This work is licensed under the Creative Commons Attribution International License (CC BY 4.0).

http://creativecommons.org/licenses/by/4.0/

(c) (i) Open Access

\begin{abstract}
Background: The number of medical lawsuits has been increasing in Japan. This research aimed to clarify trends in radiology-related cases based on a database search of lawsuits and to consider how to avoid the problems that lead to these kinds of lawsuits. Decrease of radiology-related medical lawsuits makes radiologists work more comfortable. Materials and Methods: The Japanese court case search system "D1-Law.com", a comprehensive database that includes 29,000 laws and 210,000 precedents, was used to search for relevant lawsuits. The primary $(\alpha)$ keyword was related to radiology. A search was conducted as " $\alpha$ " $\times$ "medical" $\times$ "compensation for damages" for the period from 1 January 1965 through 30 September 2013. Basic information on 3383 extracted lawsuits was examined. Of these, 35 cases directly related to radiologists were selected, and the judgments or outcomes were investigated. All cases were classified by modality (type of treatment), whether damages were awarded in each modality, and whether the litigation issues were related to procedure, informed consent (IC), diagnosis, or interpretation. Court judgments were analyzed to determine whether a causal relationship was established and how that affected the outcome. Results: The number of lawsuits gradually increased in the 1980s and 1990s and then began to decrease slightly in the 2000s. Interventional radiology (IVR) had the greatest number of cases and greatest number where compensation was awarded. No characteristic trends were identified with regard to litigation issues. There was a tendency to reject cases where no causal relationship was recognized and the treatment was considered appropriate. Conclusion: Mistakes in IVR procedures, lesions overlooked during image interpretation, and misdiagnoses were the main causes of litigation. In IVR, it is important to improve techniques and establish improved communication and trust with patients before and after therapy. In addition to developing methods to prevent overlooking lesions, the adequacy of all diagnoses obtained from radiological images must be carefully
\end{abstract}


reviewed. Results of our study require the radiologists careful and precise image interpretation and intimate relationship with the patients in addition to a certain technique when performing IVR.

\section{Keywords}

Lawsuits, Radiologist, Interventional Radiology (IVR), Image Interpretation, Misdiagnosis

\section{Introduction}

The number of medical lawsuits increased more than doubled in the 1990s compared to the1980s in Japan, after which it leveled off and then slightly decreased in the 2000s. Currently, there are about 800 new medical lawsuits each year [1] [2] [3]. The progress of CT and MRI was remarkable. The appearance of Multi detecter CT, the shooting speed became faster and the image quality improved [4]. In MRI, shooting time was shortened and image quality was improved [5]. Lawsuits related to radiology and diagnostic imaging include those where X-ray, computed tomography (CT), magnetic resonance imaging (MRI), angiography, nuclear medicine, and contrast agents were used. The aim of this study was to clarify lawsuit trends in the fields related to radiology on the basis of cases retrieved from a search of a legal database for terms related to radiology and imaging and to consider measures to avoid the problems that lead to these kinds of lawsuits.

\section{Materials and Methods}

One of the authors has a quality of the lawyer and performed the following search. The search system "D1-Law.com," a comprehensive database of legal information in Japan containing current laws and regulations as well as past and ongoing lawsuits, was used for the search. The database includes about 29,000 reviews of laws and regulations and about 210,000 precedents. It can be searched by using both full-text searches and multiple keywords.

Radiology-related keywords (Figure 1) were regarded as the " $\alpha$ " search item. Searches were conducted on 30 September 2013 as " $\alpha$ " $\times$ "medical" $\times$ "damages" for the each term. The search period was from 1 January 1965 to 30 September 2013.

\section{Results}

A breakdown of the 3383 search results by keyword is shown in Table 1. Basic information of the lawsuits was reviewed, and 35 cases were extracted in which a radiologist or radiology-related procedure was the subject of the lawsuit. Details of these 35 cases are summarized in Table 1 . The terms and results are explained in more detail below. 
Table 1. Radiology-related lawsuits, 1965-2010.

\begin{tabular}{|c|c|c|c|c|c|c|c|c|}
\hline No & Judgment & Modality & Rejection/Admit & Procedure & IC & Diagnosis & Causality & Adequate/Inadequate \\
\hline 1 & 1966.3 & IVR & Rejection & O & O & & No & Adequate \\
\hline 2 & 1968.7 & Myelography & Rejection & & O & & No & Adequate \\
\hline 3 & 1973.2 & Myelography & Rejection & & O & & No & Adequate \\
\hline 4 & 1977.1 & Myelography & Rejection & & O & & Yes & Adequate \\
\hline 5 & 1977.2 & Myelography & Rejection & & O & & No & Adequate \\
\hline 6 & 1978.7 & IVR & Rejection & O & & & No & Adequate \\
\hline 7 & 1979.4 & Myelography & Rejection & & O & & No & Adequate \\
\hline 8 & 1980.11 & Radiation therapy & Rejection & O & & & Yes & Adequate \\
\hline 9 & 1982.7 & Myelography & Admit & O & & & Yes & Inadequate \\
\hline 10 & 1983.6 & X-ray & Admit & & & O & Yes & Inadequate \\
\hline 11 & 1987.11 & $\mathrm{X}$-ray & Rejection & & & O & No & Adequate \\
\hline 12 & 1989.3 & IVR & Rejection & & O & & Yes & Adequate \\
\hline 13 & 1992.4 & BreastUS/MMG & Rejection & & & O & No & Adequate \\
\hline 14 & 1993.5 & $\mathrm{CT} / \mathrm{MRI}$ & Admit & O & & & Yes & Inadequate \\
\hline 15 & 1993.6 & X-ray & Rejection & & & O & No & Adequate \\
\hline 16 & 1994.1 & $\mathrm{X}$-ray & Rejection & & & O & Yes & Adequate \\
\hline 17 & 1994.7 & IVR & Admit & & & O & Yes & Inadequate \\
\hline 18 & 1995.1 & CT/MRI & Admit & & & O & Yes & Inadequate \\
\hline 19 & 1995.2 & Radiation therapy & Admit & O & & & Yes & Inadequate \\
\hline 20 & 1996.2 & IVR & Admit & O & 0 & & No & Adequate \\
\hline 21 & 1998.1 & $\mathrm{CT} / \mathrm{MRI}$ & Admit & & & O & No & Inadequate \\
\hline 22 & 1998.12 & IVR & Admit & O & O & & No & Inadequate \\
\hline 23 & 1998.7 & IVR & Rejection & O & & O & No & Adequate \\
\hline 24 & 1999.8 & CT/MRI & Rejection & O & & & No & Adequate \\
\hline 25 & 2000.1 & IVR & Admit & O & & & Yes & Inadequate \\
\hline 26 & 2000.2 & IVR & Admit & O & & & Yes & Inadequate \\
\hline 27 & 2000.5 & X-ray & Admit & & O & & Yes & Inadequate \\
\hline 28 & 2001.11 & IVR & Admit & & & O & Yes & Inadequate \\
\hline 29 & 2002.1 & $\mathrm{X}$-ray & Admit & & & O & Yes & Inadequate \\
\hline 30 & 2002.6 & X-ray & Rejection & & & O & No & Adequate \\
\hline 31 & 2003.2 & IVR & Admit & & O & & No & Adequate \\
\hline 32 & 2005.12 & $\mathrm{X}$-ray & Admit & & & O & Yes & Inadequate \\
\hline 33 & 2006.1 & IVR & Rejection & O & O & & No & Inadequate \\
\hline 34 & 2006.9 & IVR & Admit & O & & & Yes & Inadequate \\
\hline 35 & 2010.4 & BreastUS/MMG & Admit & & & O & Yes & Adequate \\
\hline
\end{tabular}

A case where litigation was approved is denoted as admit, and a rejected case is denoted as rejection. A case in which the action taken that caused the lawsuit was found to be correct was noted as adequate, and a case where the action was deemed inappropriate is noted as inadequate. 


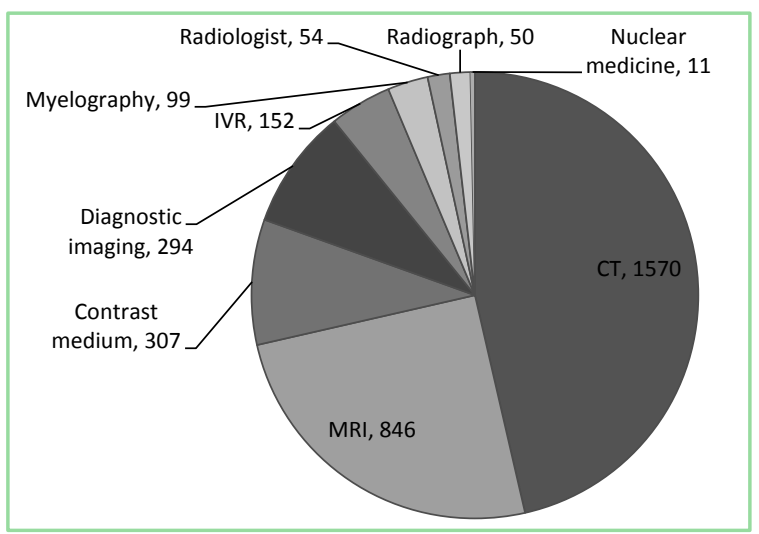

Figure 1. Radiology-related keywords and number of extracted lawsuits. The most extracted keyword was CT, followed by MRI.

1) Judgement dates and trends

The number of the radiology-related medical lawsuits gradually increased from the beginning of the study period through the 1980s and then more rapidly increased in the 1990s before flattening out and decreasing in the 2000s (Figure 2).

2) Rejections and damages in each modality

Damages were awarded in 18 of the 35 cases; the claim was rejected in the other 17. IVR was the modality with both the greatest number of cases and greatest number of damage awards (Table 2). Lawsuits were dismissed in five of six cases on myelography because causality between ethyl iodophenyl undecylate (Myodil) and headaches and neurological symptoms was not proven [6] [7]. In the sixth case, the amount of contrast medium used was clearly greater than the usage indicated in the package insert; in addition, the instructions after the examination were inappropriate. The X-ray category had eight cases, including an air-contrast enema and stomach fluoroscopy. Damages were awarded in four of the cases. Damages were awarded in three of the four cases related to $\mathrm{CT} / \mathrm{MRI}$ use, which is a small number considering the increased use of CT/MRI examinations in recent years. There were two cases in the breast ultrasound (US)/mammography (MMG) category, one with damages and one rejection.

3) Causes of lawsuits

Each lawsuit issue was classified into one of three categories related to cause: procedure, informed consent (IC), and diagnosis/interpretation [8] (Table 3). Both procedure and IC were points at issue in one case, and both procedure and diagnosis were at issue in four other cases. The totals shown in Table 3 therefore reflect 40 causes for the 35 cases.

4) Decision rationale

The rationale for each lawsuit decision was divided into the claim of the parties and the certification of the court. The latter was divided into four parts: the factual course, presence or absence of a causal relationship, appropriateness of the treatment and doctor's medical judgement, and the extent of damages. The 


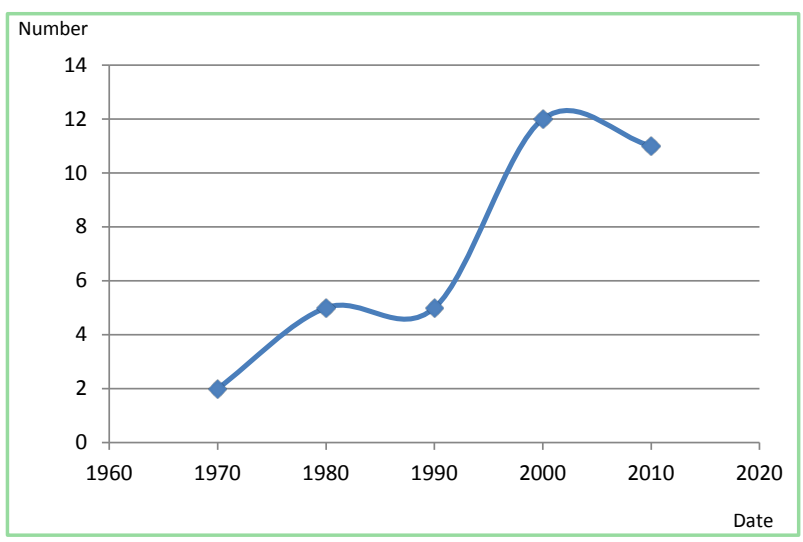

Figure 2. Sequential changes of radiology-related lawsuits. Radiology-related lawsuits have increased from 1970 to 1980 and from 1990 to 2000. Especially, increase from 1990 to 2000 was steep followed by decrease from 2000 to 2010 .

Table 2. The numbers of cases where damages were awarded or rejected in each modality.

\begin{tabular}{cccc}
\hline & Damages & Rejection & Total \\
\hline IVR & 8 & 5 & 13 \\
Myelography & 1 & 5 & 6 \\
X-ray & 4 & 4 & 8 \\
CT/MRI & 3 & 1 & 4 \\
Radiation therapy & 1 & 1 & 2 \\
Breast US.MMG & 1 & 1 & 2 \\
Total & 18 & 17 & 35
\end{tabular}

Table 3. The numbers of cases where damages were awarded or rejected by cause.

\begin{tabular}{cccc}
\hline & Damages & Rejection & Total \\
Procedure & 8 & 6 & 14 \\
Informed consent & 4 & 8 & 12 \\
Diagnosis interpretation & 8 & 6 & 14 \\
Total & 20 & 20 & 40 \\
\hline
\end{tabular}

Some cases were included in more than one category.

presence or absence of a causal relationship between the radiological test and a damage award decision (damages or rejection) was analyzed, as was the adequacy of the treatment and the doctor's medical judgement (Table 4). Damages were awarded in 18 cases.

\section{Discussion}

The number of medical lawsuits involving radiologists gradually increased in the 1970s and 1980s, rapidly increased in the 1990s, and then leveled off and decreased 
Table 4. The numbers of cases where damages were awarded by causal relationship and adequacy of measures.

\begin{tabular}{ccccc}
\hline & \multicolumn{2}{c}{ Causal relationship } & $\begin{array}{c}\text { Whether treatment and doctor's medical } \\
\text { judgement were adequate }\end{array}$ \\
\cline { 2 - 5 } & Yes & None & Adequate & Inadequate \\
\hline Damages $(n=18)$ & $14(77 \%)$ & $4(22 \%)$ & $3(17 \%)$ & $15(83 \%)$ \\
Rejection $(n=17)$ & $4(24 \%)$ & $13(76 \%)$ & $16(94 \%)$ & $1(6 \%)$ \\
\hline
\end{tabular}

slightly in the 2000s. This pattern is consistent with the trend reported by Magnavita et al. [9] [10] for Italian medical litigation and with the trend of overall medical litigation cases in Japan, which increased from 488 cases in 1995 to 1110 in 2004 and thereafter leveled off. An increase in the number of examinations was not accompanied by an increase in the number of radiologists led to more oversights and misdiagnoses, and an increased number of procedural mistakes has resulted in an increase in the number of lawsuits. The doctor-patient relationship has also changed. In the past, there was a close personal and trusting relationship between a doctor and patient in many cases, which was helpful in mitigating and suppressing patient dissatisfaction [2] [11] [12]. However, that relationship has become more impersonal, such that it has become more of a "contract," and legal disputes more readily occur when treatment results are not satisfactory [8] [13]. In addition, many patients come to radiology departments for consultations from other hospitals and departments. In this case, radiologists have limited time to build sufficient trust with patients, and a lack of communication lends itself to an increased risk of problems developing into medical lawsuits. In the second half of the 2000s, changes occurred that contributed to the decline in the number of lawsuits. Public opinion shifted toward cooperation in interactions with health care providers. Furthermore, medical institutions have begun to emphasize improved communication with patients and family members [14].

Lawsuits and damage awards have become more common in the IVR era [15] [16]. Procedural negligence and a lack of sufficient explanation before IVR were the most frequently cited causes for damage awards. According to Magnavita et al., the most common cause of IVR lawsuits is misunderstandings that arise from a lack of communication before and after the therapy [9].

For example, a man in his 60s underwent catheter coil embolization for a left internal carotid artery aneurysm. However, he died of coil deviation followed by cerebral infarction. In this case, there were three issues of dispute in the lawsuit: 1) whether there was negligence in the choice of coil embolization, 2) whether there was negligence in the coil embolization procedure, and 3) whether there was a breach of duty in the explanation of the procedure and its risks. The first two issues were rejected, and the third was accepted. Initially, the patient had been told he would receive clipping surgery entailing a craniotomy, but the surgery choice was altered by doctor conference the day before the operation. In 
short, the patient had agreed to a treatment method that had been explained one week earlier and only received a brief, 30-minute explanation of the change one day before the scheduled treatment. An angiography was then performed, and the patient died as the coil diverged. The patient's family filed a lawsuit because there was no explanation for the change in therapy from clipping surgery to coil embolization or change of treatment from coiling to craniotomy in case of difficulties with the coiling option. Moreover, they claimed the patient had not been told that death was a possible outcome. In the judgment, the violation of duty (explanation) was accepted. During informed consent, the patient has the right to choose benefits and risks for each of the selectable treatments and to choose one of them which is thought to be best for the patients [17].

This case is an example of a case developing into a lawsuit when the trust relationship with the patient became damaged, even when there had initially been a good relationship. According to Nagashima et al. if it is judged that informed consent does not reflect the right of self determination of patients, doctors tend to be negligent [18].

Myelography trials have been conducted continuously since the late 1960 and 1970s. Oily contrast agents such as ethyl iodophenyl undecylate (Myodil) were widely used in myelography at that time. These contrast agents were thought to be absorbed after the examination. However, Myodil causes both early and late disorders, at a rate of more than 60\% [6] [7]. Early disorders such as headaches, fever, and vomiting are transient in $80 \%$ of patients. About $20 \%$ of early disorders shift to late disorders, such as movement disorders and arachnoiditis, due to adhesion of the contrast medium to the spinal cord, and the symptoms last for a long time.

In the X-ray category, the judgments took into account the testimony of expert witnesses recommended by the relevant academic societies. In addition, lesions that could not be identified by more than half of the experts tended to be rejected as evidence against a doctor. The judgments also took into account information such as statistics and prevailing medical standards. Examples of cases with compensation for damages include ones where gastric cancer had been misdiagnosed as gastritis despite multiple examinations, and a pulmonary mass was misdiagnosed as pneumonia and had not been examined precisely.

In the CT/MRI category, the workload per radiologist has been growing enormously as the number of exams and the number of images per exam have increased, and the number of lawsuits for lesions overlooked during image interpretation and misdiagnoses has been increasing [1]. In cases where lesions are overlooked despite multiple exams or cases where a cancer is diagnosed as benign, the need for compensation tended to be recognized. There was one rejected case where the patient died of myocardial infarction during the CT examination, but there was no causal relationship established between the death and the CT examination [19].

In the breast ultrasound/MMG category, one reason for damages was mental distress because the patient was inappropriately treated even though multiple 
masses were found in the breast ultrasound/MMG. The patient consequently died as the tumors grew larger and metastasized, even though there had been four follow-up examinations over several years. In the rejected case, cancer metastasized to the liver, and the patient died because a $12-\mathrm{mm}$ tumor was missed in breast cancer screening. One study found 14.83 misdiagnoses per 1000 cases for MMG and that imaging examinations had the highest rate of misdiagnosis [19]. Because the sensitivity of cancer detection is as low as $70 \%-80 \%$ [20], breast ultrasound/MMG should be performed carefully.

In the court judgments, the existence of a causal relation between an adverse event and medical action was important. Judgments were also greatly influenced by whether subsequent treatments and decisions were appropriate. In one case where damages were dismissed, the explanation of the procedure-but not necessarily the treatment itself-was judged to be inappropriate. We therefore conclude that there is a tendency to reject cases where a causal relation has not been recognized and the treatment is deemed to be appropriate.

\section{Conclusion}

Radiology-related medical lawsuits generally showed the same temporal trends as medical lawsuits as a whole. In radiology, mistakes in IVR procedures, lesions overlooked when the image is interpreted, and mistakes in diagnosis are characteristic issues. In IVR, improving techniques and building better communication and trust with patients before and after the therapy is important. In addition to developing measures to prevent overlooking lesions during image interpretation, there is a need to review the adequacy of diagnoses considered from clinical findings. Decrease of radiology-related medical lawsuits makes radiologists work more comfortable.

\section{References}

[1] Lee, C.S., Nagy, P.G., Weaver, S.J., et al. (2013) Cognitive and System Factors Contributing to Diagnostic Errors in Radiology. AJR. American Journal of Roentgenology, 201, 611-617. https://doi.org/10.2214/AJR.12.10375

[2] Halpin, S.F.S. (2009) Medico-Legal Claims against English Radiologists: 1995-2006. The British Journal of Radiology, 82, 982-988. https://doi.org/10.1259/bjr/61782960

[3] Tanabe, N. (2014) Pathologist and Medical Malpractice Law Suits. Pathology and Clinical Medicine, 32, 1277-1282. (In Japanese)

[4] Justin, S., Achille, M., Rendon, C.N., et al. (2015) Quantitative Features of Liver Lesions, Lung Nodules, and Renal Stones at Multidetector Row CT Examination. Radiology, 279, 185-194.

[5] Andreas, M.L., Daniel, V.L., Manojkumar, S., et al. (2017) Increased Speed and Imagequality for Pelvic Single-Shot Fast Spin-Echo Imaging with Variable Refocusing Flip Angles and Full-Fourier Acquisition. Radiology, 282, 561-568. https://doi.org/10.1148/radiol.2016151574

[6] Davies, F.L. (1956) Effect of Unabsorbed Radiographic Contrast Media on the Central Nervous System. Lancet, 2, 747-748. https://doi.org/10.1016/S0140-6736(56)90958-8 
[7] Matsuoka, A. and Hatori, S. (1982) Cervical Vertebral Myelography (Comparison between Myodilt and Amipaque). Clinical Orthopedic Surgery, 17, 1011-1022. (In Japanese)

[8] Richard, F. (2001) Error in Radiology. Clinical Radiology, 56, 938-946. https://doi.org/10.1053/crad.2001.0858

[9] Magnavita, N., Fileni, A., Mirk, P., et al (2012) Malpractice Claims in Interventional Radiology: Frequency, Characteristics and Protective Measures. La Radiologia Medica, 118, 504-517. https://doi.org/10.1007/s11547-012-0878-7

[10] Fileni, A. and Magnavita, N. (2006) A 12-Year Follow-Up Study of Malpractice Claims against Radiologists in Italy. La Radiologia Medica, 111, 1009-1022. https://doi.org/10.1007/s11547-006-0099-Z

[11] Wu, A.W., Boyle, D.J., Wallace, G., et al. (2013) Disclosure of Adverse Events in the United States and Canada: An Update, and a Proposed Framework for Improvement. Journal of Public Health Research, 2, 186-193. https://doi.org/10.4081/jphr.2013.e32

[12] Bazzocchi, M. (2012) Doctor-Patient Communication in Radiology: A Great Opportunity for Future Radiology. La Radiologia Medica, 117, 339-353. https://doi.org/10.1007/s11547-012-0800-6

[13] Stephan, R.B., Jeremy, S., Whang, L.L., et al. (2012) The Demography of Medical Malpractice Suits against Radiologist. Radiology, 266, 539-547.

[14] Terada, A. (2011) Recent Trend in Medical Accident. NKSJ-RM Report E-11 1-9. (In Japanese)

[15] Francesco, P.B., Paola, F. and Alessandro, S. (2015) Errors and Malpractice Lawsuits in Radiology: What the Radiologist Needs to Know. La Radiologia Medica, 120, 779-784. https://doi.org/10.1007/s11547-015-0561-X

[16] Gregory, Q.H. and Smouse, H.B. (2006) Lessons Learned on How to Protect an Interventional Radiologist against Malpractice Claims. Seminars in Intervenstional Radiology, 23, 315-318.

[17] Zajdel, J., Dziki, A., Dziki, L., et al. (2017) Legal Grounds for Extending the Scope or Type of Procedure. Annals of Agricultural and Environmental Medicine, 24, 606-609. https://doi.org/10.5604/12321966.1232554

[18] Nagashima, H., Wada, Y. and Hongo, K. (2017) Trend of Malpractice Litigation against Neurosurgeons in Japan: An Analysis of Disclosed Database by Courts in Japan from 2001 through 2015. Neurologia Medico-Chirurgica (Tokyo), 57, 426-432. https://doi.org/10.2176/nmc.oa.2017-0049

[19] Jeremy, S.W., Stephen, R.B., Ronak, P., et al. (2012) The Causes of Medical Malpractice Suits against Radiologist in the United States. Radiology, 266, 548-554.

[20] Van breest, S.V., Setz-Pels, W., Groenewoud, J.H., et al. (2012) Malpractice Claims Following Screening Mammography in The Netherlands. International Journal of Cancer, 131, 1360-1136. 